\title{
LA UNIFICACIÓN DE LA CIENCIA COMO ACCIÓN SOCIAL: OTTO NEURATH Y JOHN DEWEY ${ }^{1}$
}

\author{
J. Miguel ESTEBAN \\ UAEM, México
}

Resumen: Tras el giro naturalista hacia las prácticas en la filosofia contemporánea de la ciencia parece pertinente preguntarse en qué sentido la unidad de la ciencia fue, es o puede ser un problema de significación social. En este escrito defiendo que Otto Neurath y John Dewey anticipan este giro, compartiendo una concepción holistica, social y transformativa de las prácticas cientificas orientada por el proyecto de la Ciencia Unificada, y que ya en 1938, Dewey y Neurath abrazaban el programa de unificación de la ciencia como parte de un programa de acción social. Defiendo también que es ese programa común el que explica la colaboración de un pragmatista como John Dewey en un proyecto fundamentalmente positivista como la Enciclopedia, y que la colaboración de Dewey y Neurath en el proyecto de unificación de la ciencia nos ayuda a entender de otra manera la comunidad de intereses entre el pragmatismo norteamericano y el positivismo lógico europeo. El pragmatismo de Dewey y el empirismo de Neurath eran versiones de una misma apuesta: la apuesta por la educación como principal motor del cambio social.

Palabras clave: Dewey, Neurath, ciencia unificada, naturalismo, positivismo, prácticas, acción social.

Abstract: Naturalizing turn to practices in contemporary philosophy of science leads to the problem the social significance of the unity of science. In this paper I sug-

1 Esta escrito fue presentado como conferencia ante profesores de los departamentos de Filosofía y de Lógica, Historia y Filosofía de la Ciencia de la UNED. Agradezco a todos los asistentes, y en particular a los profesores Francisco J. Álvarez, Luis Vega, y Ramón del Castillo sus sugerentes críticas y pertinentes comentarios 
gest that Otto Neurath and John Dewey are foreruners of that turn, for they share a holistic, social and transformative conception of scientific practices, a conception guided by the Unified Science Project. Both embraced this project as a part of a social action program. I also suggest that this shared program explains why Dewey collaborated in such an essentially positivistic program as the Encyclopaedia. This collaboration help us to understand many common interests shared by American Pragmatism and European Logical Positivism. Dewey's Pragmatism and Neurath Positivism are but two ways of the same bet: education as the key to social change.

Keywords: Dewey, Neurath, unified science, naturalism, positivism, practices, social action.

\section{Preliminares}

El lema de la unidad del conocimiento ha estado presente en casi toda la historia de la filosofía occidental. Su significación no siempre ha sido la misma, claro está ${ }^{2}$. En la primera mitad del siglo XX, el lema fue uno de los motores que impulsaron la constitución de filosofía de la ciencia como disciplina académica. Aún hoy resulta un tema de interés, aunque las opiniones al respecto parecen algo encontradas. Es más, simplificando un tanto las cosas, cabría afirmar que la filosofía contemporánea de la ciencia se halla bipolarizada en cuanto a la posibilidad y el sentido de la unificación de la ciencia.

Autores como Michael Friedman y Philip Kitcher ${ }^{3}$ han ligado unificación, verdad y explicación. Kitcher, por ejemplo, postula la unificación de la ciencia

2 Johannes Kepler, por ejemplo, postulaba dicha unidad sobre un principio ontológico de simplicidad, mientras que Francis Bacon la construía sobre bases principalmente metodológicas. Descartes hablaba del árbol de la ciencia o del edificio del conocimiento, combinando la aspiración a la unidad metodológica con la aspiración a una certeza epistemológica absoluta, derivada de un saber que se sabía ontológicamente distinto de su objeto. Kant construía la unidad de la razón arquitectónicamente, a partir de las condiciones a priori de todo conocimiento posible. Una vez consumada la experiencia histórica de la conciencia hacia la ciencia, Hegel sistematizaba la unidad del saber en una Enciclopedia de las Ciencias Filosóficas. Para el empirismo, el lema de la unidad de la ciencia ha venido acompañado de un motivo auxiliar: la demarcación entre ciencia y no ciencia. Este es el caso del positivismo de Comte y del empirismo lógico de la primera mitad del siglo Xx. Carnap añadia al lema de la unificación y la demarcación la idea de reconstruir la ciencia en un lenguaje universal. Algo a lo que también había aspirado Leibniz.

3 Michael FRIEDIRIAN, "Explanation and Scientific Undertanding", Journal of Philosophy 71 (1974): 5-19; Philip KUTCHER, «Explanatory Unification», Philosophy of Science 48 (1981): 507-531. 
como condición del progreso científico e incluso coquetea con la idea peirciana de La Realidad como causa final de La Ciencia:

«El crecimiento del conocimiento científico se rige por una práctica de unificación [...1 unificar nuestra explicación es para nosotros un desideratum, uno que colocamos antes de encontrar la verdad literal en las muchas ocasiones en que idealizamos los fenómenos. La estructura causal del mundo, las divisiones de las cosas en clases y las dependencias objetivas entre los fenómenos surgen de nuestros conceptos organizativos. Así, decir que un predicado particular delimita una clase natural es afirmar que determinar la extensión de ese predicado será una parte de la práctica final (ideal). Declarar que un esquema es correcto equivale a predecir que ese esquema tomará parte en la unificación ideal de los fenómenos» ${ }^{4}$.

Otro filósofo contemporáneo, Patrick Suppes, ha señalado tres tipos de reductivismo inherentes a la idea de unificación de la ciencia: reductivismo lingüístico, epistemológico y metodológico. En su opinión, además, hay una doctrina de la completud implícita en la tesis de la unidad de la ciencia:

«Esa doctrina a menudo se expresa en suposiciones acerca de la uniformidad de la naturaleza y de un universo completamente ordenado y por lo tanto de carácter perfectamente cognoscible. Es sencillo poner juntas unidad, certeza y completudy producir así una deliciosa fantasía filosófican ${ }^{5}$.

Radicalmente opuestos a esta fantasía, a la que el mismo Kitcher ha denominado la leyenda de la ciencia, se encuentran la mayoría de los teóricos de la ciencia de orientación sociológica. Peter Galison y otros han denunciado lo que Hilary Putnam llamó el fetichismo del método, algo que bien podía ampliarse al fetichismo del lenguaje ideal o la práctica ideal. Según Galison, hay diferentes prácticas científicas no organizadas jerárquicamente y en pugna por la supremacía, entendida en términos de relaciones de poder entre grupos de seres humanos ${ }^{6}$. La ciencia se asemejaría más a los pasillos o las trastiendas del

4 P. KITCHER, El Avance de la Ciencia (México: UNAM_IIF, 2000), pp. 242-243.

5 P. SUPPES, "The Plurality of Science", Proceedings of the Philosophy of Science Association, 1978, \#4.

6 Peter Galison y David StUmp, The Disunity of Science: Boundaries, Contexis and Power (SUP: Stanford, 1996). 
cabildeo político que al orden arquitectónico de la razón. La unidad de la ciencia sería una quimera propia de filósofos.

En mi opinión, el constructivismo sociológico plantea un reto al menos digno de ser tomado en consideración y nos obliga a abordar el problema de la unidad de la ciencia desde otro punto de vista. Una vez dejamos de considerar las ciencias como sistemas de enunciados para pasar a considerarlas como conjuntos más o menos difusos de prácticas, resulta necesario preguntarse en qué sentido la unidad de la ciencia fue, es o puede ser un problema de significación social. Éste era precisamente el título de la contribución de John Dewey al primer volumen de la Enciclopedia de la Ciencia Unificada editada por Otto Neurath en 1938. En este escrito me propongo defender que Dewey y Neurath abrazaban el programa de unificación de la ciencia como parte de un programa de acción social, y que es ese programa común el que explica la colaboración de un pragmatista como John Dewey en un proyecto fundamentalmente positivista como la Enciclopedia. En consecuencia, también defenderé que la colaboración de Dewey y Neurath en el proyecto de unificación de la ciencia nos ayuda a entender de otra manera la comunidad de intereses entre el pragmatismo norteamericano y el positivismo lógico europeo ${ }^{7}$.

7 El interés por las relaciones entre las distintas variantes filosóficas del empirismo y del pragmatismo nace ya con la propia gestación del pragmatismo norteamericano durante el siglo XIX. Max Fisch, por ejemplo, ha señalado el peso que la filosofía de un seguidor de John Stuart Mill, el filósofo escocés Alexander Bain, tuvo en esa gestación. De hecho, aunque Charles Sanders Peirce era muy crítico con el nominalismo empirista, su máxima pragmática se inspira abiertamente en la noción de creencia de Bain. Por otra parte, sobre la deuda de William James con el empirismo el consenso es mucho mayor. El mismo JAMES bautizó a su pragmatismo como un empirismo radical. Robert Roth, por ejemplo, señala el origen humano del tratamiento de la identidad personal en William James y el influjo de Bentham y Mill en su teoría psicológica. Por último, y pese a la gran disputa que John Dewey mantuvo con Bertrand Russell durante muchos afios, el talante empirista de la filosofia de Dewey es también notorio. En "An Empirical Survey of Empiricisms", por ejemplo, Dewey formula con una claridad inusual el carácter empírico de su filosofía. Por lo demás, su Logic: The Theory of Inquiry entronca con una tradición metodológica que se remonta a Mill y a Bacon. En el siglo xx las relaciones entre el empirismo y el pragmatismo continuaron, pero fueron los empiristas lógicos quienes dirigieron su mirada hacia el pragmatismo norteamericano. Aunque nacido en Viena, el empirismo lógico alcanzó su apogeo en el segundo tercio del siglo pasado en los Estados Unidos, debido en parte al exilio de pensadores como Hans Reichenbach y Rudolf Carnap. W.V. Quine y Ch. Morris, formados aún en una tradición pragmatista aún hegemónica en Norteamérica hasta los ańos treinta, habían acudido al círculo empirista europeo para realizar sus estudios posdoctorales. Las aportaciones teóricas de Quine y Morris resultaron cruciales para la recepción norteamericana del empirismo, sobre por su interpretación del llamado giro lingütstico. De hecho, los estudios clásicos sobre 
Dewey sentía un gran aprecio personal e intelectual por Neurath ${ }^{8}$. Creía sinceramente que Neurath era el único miembro del positivismo lógico que se había aproximado convincentemente hacia al pragmatismo, alejándose de la concepción fundacionalista de la ciencia compartida por otros miembros del movimiento positivista. Frente a esta concepción, Neurath y Dewey comparten una idea holística, social y transformativa de las prácticas científicas mucho más próxima a las concepciones naturalistas contemporáneas, como las de Ian Hacking o Ronald Giere. En lo que resta intentaré mostrar cómo la atención que ambos autores prestan a la práctica científica efectiva apunta a una concepción naturalista de la filosofía de ciencia cuyo leit motiv es un programa común de acción social. En el primer epígrafe (I) analizaré ese componente

la absorción del pragmatismo por parte del empirismo lógico se han centrado principalmente en el criterio empirista de significación cognitiva, derivado de una interpretación de la máxima pragmática de Peirce en términos estrictamente semánticos. La percepción de las consecuencias del criterio empirista de significación cognitiva para la propia actividad filosofica imprimió un nuevo giro a las relaciones entre el pragmatismo y el empirismo. En 1951 Quine denunciaba dos dogmas de empirismo vinculados con el criterio de significación: la analiticidad, la verdad en virtud puramente del significado, y el reductivismo, la verificación entendida en términos atomistas. Según Quine, adoptar un pragmatismo más completo implicaba abandonar ambos dogmas y desdibujar las fronteras entre ciencia y filosofia que el empirismo lógico había trazado. En las décadas siguientes, un discípulo del positivista Hans Reichenbach, Hilary Putnam, se pasa a las filas del pragmatismo, denunciando al empirismo lógico como la gran manifestación intelectual del impacto de la ciencia en las concepciones modernas de la racionalidad, basadas en su opinión en la dicotomía hecho/valor. En esos años también , Paul Feyerabend se había atrevido a preguntar "¿Como ser un buen empirista?" tras haber renunciado a la racionalidad positivista. Por último, en los últimos años, una de las consignas del neopragmatismo de Richard RORTY es una pregunta para la que él presupone una respuesta negativa: «Queda algo digno de salvar en el empirismo?" Creo que el pragmatismo clásico, y en particular la obra de John Dewey, constituye una primera respuesta a la pregunta por el valor filosófico del empirismo. En estas páginas avanzo una interpretación pragmatista del empirismo a partir de las relaciones entre las filosofías de la ciencia de Otto Neurath, el empirista lógico que tanto influyera en Quine, y de John Dewey.

8 Por otra parte, la correspondencia de Dewey nos revela el poco mérito que atribuía a la filosofía de Rudolf Carnap, el otro editor europeo International Encyclopedial of Unified Science: Con el tiempo, CARNAP abandonó el proyecto y pidió que su nombre fuese formalmente retirado como editor. Lo cierto es que CARNAP concebía la unificación de manera significativamente distinta a la de Neurath. Unificar la ciencia implicaba para Carnap recontruir racionalmente las teorías científicas siguiendo las directrices del programa logicista y, en particular, de los Principia Mathematica de Whitehead y Russell. Así como Russell perseguía la fundamentación de la matemática reduciéndola a lógica más teoría de conjuntos, Carnap pretendía fundamentar las ciencias no-formales reduciéndolas a lógica más enunciados atómicos de contenido sensorial. 
naturalista en las respectivas filosofías de la ciencia de Dewey y Neurath. En el segundo (II) examinaré cómo ninguno de los dos concibe la unidad de la ciencia sin el concurso del lenguaje ordinario y las concepciones del sentido común. En el tercero (III) trataré de vincular a Neurath y a Dewey con el llamado viraje hacia las prácticas de la epistemología contemporánea. Por último, en (IV) analizaré por qué para ambos la unificación de la ciencia no es tanto un problema semántico o epistemológico, sino un proyecto de significación social. Como ya adelantaba, tener presente ese proyecto quizá pueda ayudamos a reconstruir las relaciones entre pragmatismo y empirismo. El pragmatismo de Dewey y el empirismo de Neurath eran versiones de una misma apuesta: la apuesta por la educación como principal motor del cambio social.

\section{Naturalismo}

En un libro magnífico publicado muy pocos años atrás, Nancy Cartwright, Jordi Cat, Lola Fleck y Thomas Uebel ${ }^{9}$ han defendido que el naturalismo de Neurath significó el primer paso hacia la superación del positivismo desde dentro que Quine consumara años después. Mi lectura de los textos de Neurath sigue muy de cerca la que Cartwright y su equipo realizan, si bien atribuye más relevancia al pragmatismo de Neurath (al que los autores conceden una presencia más bien limitada) y a las grandes semejanzas con la filosofía de Dewey (autor que aparece sólo un par de veces en todo el libro).

Cualquier lector atento de las obras de Neurath y Dewey puede encontrar sólidos argumentos para transformar el empirismo en naturalismo: una concepción del conocimiento alejada del modelo del espectador, es decir, del modelo clásico de la ausencia de presuposiciones ejemplificado por la tabula rasa. Uno y otro aconsejan el franco reconocimiento de la contingencia de nuestro conocimiento. Para ambos, el conocimiento depende de la oportunidad y la fiabilidad de nuestras elecciones y no de una causa final que asegure la convergencia de éstas. Pero, en su opinión, lo más que podemos hacer para asegurar la fiabilidad de nuestras elecciones es cooperar: interactuar con el entorno y con otros grupos de seres humanos que forman parte del mundo y favorecer el control público de los resultados.

9 N. CARTWRIGHT et al: Otto Neurath: Philosophy between Science and Politics (Cambridge: Cambrige University Press, 1996). 
El naturalismo de Neurath y de Dewey significa una radical desvinculación del proyecto epistemológico clásico, el que aspira a la certeza incondicionada o extramoral, sea mediante la aplicación metódica de la duda a todo contenido mental (Descartes), la disolución del conocimiento adquirido en un conjunto de sensaciones últimas imprimidas sobre una tabula rasa (Locke), o la epokhé $o$ reducción fenomenológica (Husserl), entendida como la suspensión de la actitud cognitiva natural: la puesta entre paréntesis de todo contenido en nuestras creencias que refiera a sus objetos como existencias. En la "Naturalización de la Epistemología", W.V. Quine ha entroncado la filosofía de la ciencia de un positivista como Carnap con esta tradición epistemológica, mientras que adjudica su ruptura a Neurath, otro positivista. Veamos por qué.

Carnap rechaza la propuesta de utilizar el conocimiento ya adquirido para validar el conocimiento, una propuesta tradicionalmente puesta bajo la amenaza de circularidad. Parecería que si la meta de la epistemología es fundamentar la validez de la ciencia empírica, no es posible emplear los propios resultados de la ciencia para validar esos fundamentos. Para el naturalista, por el contrario

«estos escrúpulos contra la circularidad tienen escasa importancia una vez hemos cesado de soñar en deducir la ciencia a partir de observaciones. Si lo que perseguimos es, sencillamente, entender el nexo entre la observación y la ciencia, será aconsejable que hagamos uso de cualquier información disponible, incluyendo la proporcionada por estas mismas ciencias cuyo nexo con la observación estamos tratando de entender" ${ }^{10}$. "Al contrario que Descartes", afirma Quine "contamos con nuestras creencias del momento y las usamos, incluso sumidos en el filosofar, mientras que no las cambiemos aquí y allá por algo mejor».

El teórico contemporáneo Ronald Giere ha situado el viraje naturalizado de la filosofía contemporánea de la ciencia en la obra de Kuhn ${ }^{11}$. La Estructura de las Revoluciones Cientificas, sobre todo por el papel concedido a la historia de la ciencia y por su relativización de la dicotomía entre contexto de descubrimiento y contexto de justificación. En mi opinión, aún siendo esto cierto,

10 W.V. QUINE: “La Naturalización de la Epistemología”, en La Relatividad Ontológica y otros ensayos (Madrid: Tecnos, 1974). p. 111 y Palabra y Objeto (Barcelona: Labor, 1968), p. 38.

1 Ronald Giere, La Explicación de la Ciencia, (México: CONACYT, 1992), pp. 55-56. 
el naturalismo de Kuhn se ve mermado por la atención predominante a la teoría como unidad de análisis de la ciencia ${ }^{12}$. Y aquí también el naturalismo pragmático de Dewey y Neurath ofrece cierta ventajas.

La Lógica de John Dewey ejemplifica perfectamente un tipo de filosofía naturalizada de la ciencia que concede un papel rector a las prácticas efectivas en la formación de la normatividad. Las normas lógicas surgen en contextos heurísticos a partir de un conjunto de operaciones destinadas al control de la investigación, de manera que ésta pueda deparar juicios o aserciones garantizadas. Dewey afronta directamente el reto de la circularidad:

"La investigación, si quiere alcanzar conclusiones válidas, tiene que satisfacer ciertas exigencias lógicas. Y es fácil inferir de este hecho la idea de que las exigencias lógicas se imponen desde fuera» a los métodos de investigación [..]pues se preguntará: ¡cómo es posible que la investigación, que ha de ser ponderada por referencia a un criterio, sea a su vez fuente de ese criterio?" (LW. 12.17) ${ }^{13}$.

Dewey piensa que el estudio histórico de la ciencia aporta razones para defender que la investigación científica ha sido capaz de desarrollar en su propia mar-

12 Aunque esto no es del todo cierto, claro está. El papel de las prácticas en los paradigmas ya aparece prefigurado, por ejemplo, en la comparación de ciertas partes del adiestramiento científico con los ejercicios musicales de dedos. Véase "La función del Dogma en la Investigación Científica", (Valencia: Teorema, 1980, p. 10). En "Os mapas como práticas de representação na ciencian, en Waldmiro Filho (ed.) Epistemologia e ensino de ciencia (Arcadia: Universidad do Federal de Bahía, 2002) he comentado también el empleo que, en la Estructura de las Revoluciones Cientificas, T.S. Kuhn integra ese conjunto de decisiones previas en el concepto de paradigma, empleando también y significativamente la analogía del mapa: "[...] los paradigmas no sólo proporcionan a los cientificos mapas sino también algunas de las indicaciones principales para el establecimiento de mapas. Al aprender un paradigma, el cientifico adquiere al mismo tiempo teoria, métodos y normas, casi siempre en una mezcla inseparable» Th. S. KUHN, La Estructura de las Revoluciones Cientificas (México:FCE, 1975), p. 174 Aunque Kulm parece hacer hincapié en la teoría, esta indicación sobre las directrices para levantar mapas parece acercarlo a la posición que mantendría más tarde en sus "Second Thoughts on Paradigms", donde adscribe a los ejemplos de prácticas compartidos un papel central en la actividad cientifica.

13 Los textos de John Dewey citados en estos ensayos corresponden a la edición crítica de su obra completa publicada por la Southern Illinois University Press, bajo la dirección editorial de Jo Ann Boydston: The Early Works, 1882-1898, 5 volúmenes; The Middle Works, 1899-1924, 15 volúmenes: The Later Works, 1925-1953, 15 volúmenes. Citamos con la abreviatura (EW, MW, LW) seguida por el volumen y la paginación en la edición crítica. MW6:78, por ejemplo, indica John Dewey, The Middle Works, volumen 6, p. 78. 
cha criterios y normas a las que se han sometido posteriores investigaciones. En este sentido la práctica de la ciencia no es diferente de otras actividades autocorrectivas de los organismos humanos, desde las artes industriales hasta el derecho. El hecho de que las normas hayan sido formadas no las incapacita para ser formativas. Dewey plantea una analogía con las formas jurídicas que resulta iluminadora para entender la interacción entre lo fáctico y lo normativo.

"Los materiales de las regulaciones jurídicas son transacciones que ocurren en los seres humanos y grupos de seres humanos; transacciones de un cierto tipo que se entablan aparte de la ley. Cuando ciertos aspectos y fases de estas transacciones se hallan legalmente formalizados, surgen conceptos tales como contravenciones, delito, daños, contrato,etc. Estas concepciones formales surgen de transacciones corrientes, no son impresas en la investigación desde arriba o desde una fuente externa o a priori. Pero una vez formados son también formativos; regulan el comportamiento adecuado de las actividades de las cuales surgieron [...] las normas en cuestión no son ni fijas ni eternas. Cambian, aunque por lo general muy lentamente, con el cambio de las transacciones habituales en que se traban individuos y grupos y con el cambio que tiene lugar en las consecuencias de estas transacciones" (LW.12..105-106).

$\mathrm{Al}$ señalar la interdependencia entre normatividad y contexto heurístico, el naturalismo normativo convierte a la filosofía de la ciencia en una disciplina social. Y también en este punto, como veremos, Dewey y Neurath estaban básicamente de acuerdo.

Resulta significativo que, en el proceso de redacción de Knowing and the Known (1944), coescrito con Arthur Bentley, Dewey sugiriera a éste la conveniencia de una nota que desmarcase a Neurath de las concepciones formalistas de la lógica que el libro crítica y que sugiera su proximidad al naturalismo lógico-normativo de Dewey:

"Si Otto Neurath hubiese vivido para desarrollar su posición más de lo que lo hizo, sin duda habría que agruparlo junto con los naturalistas. Suposiciones al respecto eran mucho más avanzadas que las de otros compañeros más activos del proyecto de la Enciclopedia Internacional de la Ciencia Unificada, de la cual él era el editor» (LW. 16.187).

Pasemos ahora a examinar el naturalismo de Neurath en sus puntos comunes con el naturalismo de Dewey. 
El mismo Quine ha remitido la concepción naturalista de la ciencia a la célebre imagen del barco de Neurath: la empresa científica como un barco que ha de ser reparado plancha a plancha en mar abierto, sin dique seco en el que varar. Cartwright, Uebel et al. han logrado distinguir distintas versiones de esta metáfora en el desarrollo de la obra de Neurath. No examinaré todas y cada una de estas formulaciones, sino sólo las más pertinentes para el tema que nos ocupa.

Como señala Cartwright, las primeras versiones del barco como metáfora de la empresa científica aún comportan el ideal de integración teoreticista de la ciencia. La unificación buscada es la unificación de una macroteoría reductiva, entendida como la representación física del mundo. El proyecto conserva un reconocible tono comtiano por cuanto se esfuerza en interrelacionar las diferentes teorías científicas en un sistema desprovisto de toda especulación metafisica. El nivel de integración ha de ser el de una especie de superciencia: la física como ciencia universal, como reza el título de una de las obras de Carnap ${ }^{14}$. El barco de Neurath es la concepción científica del mundo, tal y como Hahn, Carnap y Neurath titularon en 1929 al manifiesto positivista del Círculo de Viena. La lógica formal es el instrumento de la unificación sistemática.

Pero en 1934 Neurath ya ha dejado de creer en la unificación lógica del sistema:

"El sistema es la gran patraña cientifica. El progreso de la ciencia consiste, por así decirlo, en cambiar constantemente la maquinaria y avanzar sobre la base de nuevas decisiones. El resultado es una unidad a largo plazo que no puede derivarse lógicamente» ${ }^{15}$.

La unificación se consigue sobre la marcha, partiendo del conocimiento con el que de hecho contamos en cada momento. En 1937 Neurath ha abandonado por completo el ideal sistemático, sustituyéndolo por un proyecto enciclopédico en el que la unificación aparece como una propuesta

"No poseemos ninguna base firme absoluta desde la que levantar las ciencias. Nuestra verdadera siruación es como si estuviéramos a bordo de un barco en mar abierto y tuviésemos que cambiar distintas partes del barco durante el

14 Véase R. CaRnaP: The Unity of Science

15 Otto Neurath: "Unity of Science as Task", en Philosophical Papers 1913-1946 (Cambridge. Cambridge University Press, p. 116). 
viaje . No tenemos ninguna base absoluta para la ciencia; y nuestras discusiones sólo pueden determinar si los enunciados científicos son aceptados por cierta cantidad de científicos y de otros seres humanos. Las nuevas ideas pueden comprarse con aquellas históricamente aceptadas por las ciencias, pero no con un criterio inalterable de verdad [...] este enfoque se opone radicalmente a cualquier concepción que emplee la noción de el sistema de la investigación científica. [...] Ya que no podemos comparar las ciencias históricamente dadas con la "ciencia real", lo más que podemos hacer en nuestro trabajo científico parece ser una enciclopedia, construida cooperativamente por científicos que estén interesados en el empirismo científico. Este programa podría llamarse enciclopedismo»" ${ }^{16}$.

Dewey demuestra la misma reticencia al empleo de una noción preconcebida de sistema en el programa de unidad de la ciencia.

"[Se sigue que] un movimiento en pro de la unidad de la ciencia no necesita y no debería establecer de antemano una plataforma de aceptación obligatoria. Es esencialmente un movimiento cooperativo, de manera que los que las ideas y los puntos comunes concretos deben emerger de los mismos procesos de cooperación. Intentar formularlos de antemano e insistir en que todos los acepten es obstruir la cooperación y falsear el espíritu científicon (LW. 13.275)

En "International Science and its Encyclopedia", Neurath confiesa adherirse a la filosofía del sentido común y a la teoría de la experiencia del pragmatismo de Dewey. Como hemos visto en este epígrafe, este reconocimiento de Neurath expresa una deuda más profunda con el pragmatismo norteamericano: su naturalismo, entendido como la admisión de la inevitable contingencia de cualquier proyecto de fundamentación epistemológica. En el siguiente epígrafe examinaré cómo, en Dewey y Neurath, esa contingencia implica la presencia de conceptos y creencias históricamente formados y operantes tanto en el sentido común como en la ciencia.

\section{Los Protocolos y el Sentido Común}

Dewey concebía la ciencia en términos de un continuo proceso de reconstrucción de los materiales de la experiencia. $Y$ de hecho, para Dewey la activi-

16 Otto Neurath, "Unfied Science and its Enciclopedia", en Philosophical Papers 19161945 (Cambridge. Cambridge University Press, p. 181). 
dad transformativa de la ciencia es uno de los procesos por lo que producimos significado en la experiencia. Los materiales de los que parte la ciencia no son otros que los del sentido común. Pero el sentido común no es una entidad fija y constante, y uno de los motivos de su historicidad es precisamente su interacción con la ciencia:

«Los objetos y procedimientos científicos surgen de los problemas y métodos directos del sentido común, de los usos y los goces prácticos, y repercuten en estos últimos en una forma que refina, expande y libera los contenidos y los agentes a disposición del sentido común» (LW.12.81).

Para esa producción de significado resulta necesario el distanciamiento crítico ${ }^{17}$ de la situación cualitativa de uso y disfrute, propia del sentido común, que originó la investigación. El lenguaje cuantificacional, preciso y abstracto de la matemática contribuye en buena medida a ese distanciamiento. Pero sería un error pensar que el distanciamiento es absoluto y definitivo. Y Neurath también está perfectamente de acuerdo con Dewey en este punto: el sentido común está siempre a bordo del barco

«No hay tabula rasa. Somos como marinos que tiene que reconstruir su barco en mar abierto, sin dique seco en el que varar para reconstruirlo a partir de sus mejores componentes. [...]. Hay amasijos verbales imprecisos [Ballungen] que siempre son parte del barco. Cuando disminuimos la imprecisión en un lugar, bien puede reaparecer en otro en una medida aún mayor" ${ }^{18}$.

La concepción de Dewey y Neurath resulta así diametralmente opuesta a la de Carnap. La visión carnapiana de la unidad de la ciencia se basaba en la idea de reforma completa del lenguaje ordinario: la adopción de la lógica simbólica como lenguaje unificado de la ciencia, el instrumento para fijar definitivamente los significados. Dewey siempre se opuso a esta idea. Pero también Neurath,: en su opinión, no hay nada parecido a una fijación definitiva de los significados. Cartwright ha señalado este punto con sumo acierto:

17 En ese distanciamiento, según Dewey, ha de enmarcarse la debatida tesis de la neutralidad axiológica de la ciencia.

18 Otto NeURATH, "Trotocol Statements", en Philosophical Papers 1913-1946 (Cambridge. Cambridge University Press, 1983, p. 92). 
"[Según Neurath], no podemos impedir que haya formulaciones imprecisas. Pensar en su exclusión es metafísica. No podemos pasar por alto las aglomeraciones del lenguaje ordinario y proceder con el cálculo lógico como modelo de conocimiento. [Al hacerlo] negamos el rasgo más característico del significado: su condición de asunto social» ${ }^{19}$.

Reconocer que nuestro conocimiento y nuestro lenguaje tiene una radicación socio-histórica y contingente equivale a reconocer que lo que cuenta para la conformación de la unidad de la ciencia son las prácticas efectivas, y no ficciones filosóficas como el solipsismo metodológico de Carnap.

Neurath entiende las prácticas científicas como procesos sociales que se producen en el seno de laboratorios y otras comunidades de investigación. Cuando nuestro interés se centra en esas prácticas y no en la reelaboración lógica, los enunciados básicos o protocolares adquieren una nueva dimensión. Según Neurath, los reportes de datos y resultados tal y como se producen en esas comunidades revelan la ubicuidad de esos amasijos y aglomeraciones del lenguaje ordinario que en la práctica resultan ineliminables. Carnap, por el contrario, pensaba que esa eliminación era posible:

"Un protocolo primitivo bien entendido debe excluir cualesquiera enunciados obtenidos indirectamente, sea por inducción o de otra manera, y postula por lo tanto una distinción nítida (teórica) entre la materia prima de la investigación científica y su organización” ${ }^{20}$.

En uno de los capítulos del célebre debate sobre las oraciones protocolares del Círculo de Viena, Neurath acusa precisamente a Carnap de intentar introducir un tipo de protocolo atómico pidiendo una estricta distinción entre el hacer el protocolo y el procesamiento de los enunciados en el proceder científico. Según Carnap, ello puede lograrse si no recogemos en el protocolo ningún enunciado obtenido indirectamente. Para Neurath, la condición que Carnap impone es imposible de cumplir. Los datos reales de la práctica científica no son ficciones como los qualia sino enunciados formulados en parte en lenguaje ordinario y que recogen aglomeraciones o amasijos de convicciones sobre aspectos del comportamiento de los seres físicos. Los protocolos contienen inevita-

19 N. CARTWRIGHT et al, o.c., p. 158

20 R. CARNAP: The Unity of Science. 
blemente haces conceptuales que por el momento consideramos no-problemáticos, pero que podrían hacer muy imprecisas nuestras predicciones.

La concepción deweyana de los enunciados básicos es singularmente parecida ${ }^{21}$ a la de Neurath. En primer lugar, la distinción entre proposiciones fácticas y proposiciones conceptuales es funcional y no teórica. La selección de datos significa ya una reorganización de la experiencia. La atomicidad y la inmediatez son cualidades funcionales de los enunciados y no signos de simplicidad epistemológica tout court:

«Existe continuidad en la investigación. Las conclusiones alcanzadas en una investigación se convierten en medios, tanto materiales como de procedimiento, para llevar a cabo investigaciones ulteriores. En estas últimas se adoptan y utilizan los resultados de investigaciones anteriores sin que sean sometidos de nuevo a examen [...] existen objetos conceptuales y perceptivos establecidos y confirmados de tal suerte en el curso de diferentes investigaciones que representaría una pérdida de tiempo y derroche de energías que en investigaciones ulteriores los sometiéramos a examen antes de proceder a su utilización. Fácilmente se confunde este empleo inmediato de objetos, que nos son conocidos en virtud de una mediación previa, con el conocimiento inmediato" (LW.12.161).

Este interpretación funcional de la irrevisabilidad de algunos enunciados es bastante afín a la que Ludwig Wittgenstein defendía en Sobre la Certeza, un libro de tono fundamentalmente pragmatista. La investigación debe detenerse en algún punto: allí donde, según la célebre metáfora de Wittgenstein, nuestra pala se retuerce. Pero el límite de la justificación de la evidencia no está en ciertas proposiciones nos parezcan verdaderas de forma inmediata, como si fuera una especie de ver por nuestra parte; por el contrario, nos dice Wittgenstein, «es nuestra actuación la que subyace en el fondo del juego del lenguaje" (\&204). Considerar incuestionables ciertas proposiciones forma parte de nuestras prácticas de investigación (\&151). Ninguna de nuestras creencias tiene una propiedad intrínseca o inmediata a la que se deba nuestra certe-

21 Ernest Nagel cuenta una simpática anécdota sobre cómo Neurath logró vencer las reticencias de Dewey a participar en la Enciclopedia. Dewey quería estar convencido de que Neurath no defendía la existencia de enunciados protocolares à la Carnap. Como Neurath casi no hablaba inglés, Dewey hubo de contentarse con que el alemán le jurase que no creía en enunciados protocolares. 
za de su verdad. La bivalencia de algunas de nuestras proposiciones se sostiene por un conjunto de creencias básicas no fundamentadas que operan en nuestros juegos de lenguaje. Neurath denominaba Ballungen a ese conjunto o aglomerado de creencias imprecisas que entran en la construcción de nuestros juicios. Dewey ofrece una caracterización más disposicional estas creencias y las remite directamente a lo que llama conjoint activities, las prácticas cooperativas que garantizan tanto el acuerdo como el desacuerdo en nuestros juicios. Son los ta pragmatá, tal y como como las llamaban los griegos.

\section{La acción de conocer}

Llegamos así a un último punto de convergencia entre Dewey y Neurath. Para ambos, el conocimiento no es distinto de otras acciones humanas. Dewey mantiene una concepción abiertamente encarnada del conocimiento. El conocimiento es investigación y la investigación es respuesta del organismo cultural humano a una indeterminación objetiva en sus relaciones con el medio. En este sentido, Dewey se enfrenta a lo que Charles Taylor ha denominado desvinculación epistemológica: la concepción del conocimiento como construcción de representaciones independientemente de nuestra radicación como agentes en el mundo. De ahí que el fundacionalismo fracase puesto:

"que lo que subyace a nuestras representaciones del mundo —el tipo de cosas que formulamos en proposiciones declarativas- ya no es una representación, sino una cierta captación del mundo que tiene que ver con nuestra condición de agentes en él» ${ }^{22}$.

Tampoco para Neurath nuestra condición como sujetos cognoscentes es esencialmente distinta de nuestra condición de agentes. No hay un conjunto de reglas algorítmicas que rijan el dominio del conocer frente a un conjunto de máximas prácticas de uso aconsejable en el dominio de la acción. En un artículo temprano significativamente titulado «Los Viajeros Extraviados de Descartes" (1913), Neurath expone lo que quizá sea el primer abandono del prescriptivismo metodológico estricto que dominó la filosofía de la ciencia del siglo XX al menos hasta Kuhn. En este artículo, Neurath hace un espléndido

22 Charles TAYLoR: La Fuentes del Yo (Barcelona: Paidós, 1998), p. 32. 
análisis de un conocido texto del capítulo tercero del Discurso del Método en el que Descartes contrapone razón dura y razón blanda ${ }^{23}$, esto es, la razón regida por las reglas del método en el ámbito de la teoría o y lo que podríamos llamar las máximas provisionales y contextuales de la moral.

En el pensamiento, según este punto de vista, el curso de los acontecimientos no depende de las decisiones que tomemos. En la acción sí, incluso cuando la decisión sea no actuar. En el ámbito del pensamiento es posible suspender el juicio hasta obtener certeza. Por el contrario, la acción, según Descartes, es perentoria, no admite mayor demora o dilación y no puede ser suspendida. Es la capacidad de suspender el pensamiento lo que excluye a éste del ámbito de la acción. Neurath discrepa:

«Pero hay series enteras de ocupaciones que son semejantes al pensamiento. Por ejemplo, podemos interrumpir la construcción de una casa. Sin embargo, el tiempo más oportuno y favorable para construir la casa puede quedar atrás mientras dudamos, y la parte de la casa que ya ha sido parcialmente finalizada puede sufrir las consecuencias" ${ }^{24}$.

En el ámbito de la techné, resulta ridículo poner entre paréntesis el complejo de herramientas y saberes técnicos aprendidos a la hora de emprender una tarea. Ni siquiera Robinson Crusoe empezó de cero. Tampoco en el ámbito de la acción es imposible sustraerse a un conjunto heredado de disposiciones a la acción que integran vagamente el sentido común o la mentalidad de

23 "Mi segunda máxima consistía en ser lo más firme y resuelto que pudiese en mis acciones, y no seguir con menos constancia las opiniones más dudosas, una vez que me hubiese determinado a ello, que si hubiesen sido muy seguras, imitando en esto a los viajeros que, habiéndose extraviado en un bosque, no deben errar dando vueltas, ahora por un sitio, ahora por otro, no menos todavía detenerse en un lugar, sino caminar siempre los más derechamente que puedan en una misma dirección, sin cambiar ésta por débiles razones, cuando al principio haya sido quizá, sólo el azar el que los resolvió a elegirla; pues, de esta manera, si no van justamente donde deseaban, al menos llegaran a alguna parte, donde verosímilmente estarán mejor que en medio de una selva. Del mismo modo, puesto que muchas veces las acciones de la vida no admiten mayor demora, es verdad muy cierta que si no esta en nuestro poder discernir las mejores opiniones, debemos seguir las más probables. Y aunque no encontremos mayor probabilidad en unas que en otras, debemos, no obstante, decidimos por algunas y considerarlas después, no ya como dudosas, en cuanto se refieren a la práctica, sino como muy verdaderas y ciertas, puesto que la razón que nos ha llevado a ellas lo es".

24 Otto Neurath, "Descartes Lost Wanderers and the Auxiliary Motive", en Philosophical Papers, 1913-1946 (Dordrecht: Reidel, 1983), p. 2. 
una situación histórica, por más que esas disposiciones pertenezcan más a una dimensión tácita que explícita. ¿̨or qué pensar entonces que en el ámbito de la teoría es posible la tabula rasa, rebobinado el pensamiento para empezar todo desde cero? Tampoco en la teoría parece posible ignorar un conjunto de reglas provisionales y de conceptos e históricamente formados sobre los que se asienta cualquier nueva investigación. El error cartesiano, según Neurath, fue creer que sólo en el ámbito de la práctica son imprescindibles estas reglas preliminares y provisionales: olvidar que pensar también era una práctica

Para Dewey. Tanto teoría, como práctica y técnica involucran cognición, en el sentido amplio de resolución de problemas. En este sentido, no cabe sino admitir que Dewey sucumbió al fetichismo del método. Pero quizá sea más correcto hablar, como sugiriera Larry Hickman, de un complejo de métodos en Dewey ${ }^{25}$, o quizá de un acopio histórico de todo tipo de herramientas. Sea como fuere, sería un error concebir esa resolución de problemas en términos estrictamente algorítmicos. Antes al contrario, pese al innegable atractivo que para él tenía el método de las ciencias naturales, en su descripción del proceder científico Dewey reserva una función indispensable a la razón blanda o deliberativa. En primer lugar, en su Lógica., Dewey identifica los juicios que involucran valoración con los juicios prácticos. Todos los juicios de la práctica constituyen según Dewey valoraciones,

"puesto que les atañe juzgar sobre lo que hay que hacer a base de las ponderadas consecuencias de condiciones que, por ser existenciales, serán operantes en cualquier caso" (LW12:175).

En segundo lugar, no hay investigación científica que no involucre estos juicios valorativos:

"un punto aún más importante para la teoría lógica es que tales juicios valorativos llegan a formar parte de todos los juicios últimos. No hay investigación que no suponga juicios prácticos. $\mathrm{El}$ investigador científico tiene que ponderar constantemente la información recogida por sus propias observaciones y por los hallazgos de otros; tiene que sopesar su significación en cuanto a los problemas que habrá de abordar y a las actividades de observación, experimentación y cálculo" (LWI 2:175).

25 Larry HiCKIRIAN: Philosophical Tools for Technological Culture (Bloomington: Indiana University Press, 2000), capítulo 1. 
Y más adelante:

"La conclusión que se desprende es que las valoraciones, en su condición de juicios prácticos, no constituyen una clase separada de juicios en el sentido que pudieran enfrentarse a otras clases, sino que representan una fase inherente al juzgar mismo". (LW12: 179) ${ }^{26}$.

Esta redefinición de las relaciones entre teoría y práctica forma parte de una redefinición más amplia del conocimiento con la que tanto Neurath como Dewey mantenían un firme compromiso - un compromiso que abandona el terreno de lo puramente epistémico para adentrase en el ámbito de lo sociopolítico-. Uno y otro pensaban que para que la ilustración pudiese cumplir el ideal de emancipación era imprescindible naturalizar nuestra noción de conocimiento, concediendo un lugar central a su condición de acciones en el mundo. La ciencia es un instrumento de transformación social. La necesidad de una ciencia unificada corresponde para ambos a la necesidad de coordinar cooperativamente la acción social en un mundo planetariamente unificado.

\section{Ciencia Unificada para un Mundo Unificado}

Nancy Cartwright, Thomas Uebel y su equipo han defendido que para Neurath la unidad de la ciencia cumplía la función de caballo de Troya del socialismo. La hipótesis resulta muy sugerente y permite entender mejor la participación de John Dewey en el proyecto enciclopédico de Otto Neurath. Seguiremos esta hipótesis indicando en primer lugar cómo la concepción naturalizada del conocimiento que acabamos de examinar se plasma en su crítica a

26 Dicho de otra manera: todos los juicios racionalmente formados implican valoraciones. La diferencia es tan sólo una cuestión de grado. En ciertos casos, cuando la situación problemática que origina el juicio urge una ponderación directa de sus elementos como medios o recursos u obstáculos, y también de las consecuencias posibles en cuanto fines a perseguir, el aspecto valorativo sería el dominante. En este caso tendríamos juicios que bien pudiéramos llamar de valor en un sentido relativo, esto es, a diferencia de otros juicios en los que el componente valorativo resulta subordinado. "Pero como en todo juzgar nos encontramos con la selección de existencias para que sirvan como datos del sujeto y de ideas para que sirvan como posibilidades de predicación (o fines en perspectiva) resulta que la operación de valorar es inherente al juzgar como tal. Cuanto más problemática sea la situación y más penetrante la investigación con que hay que abordarla, tanto más explícita resulta la etapa valorativa» (LW12:18 l). 
algunas versiones del socialismo marxista —al que, por otra parte-, ambos se hallaban muy próximos.

Aunque Neurath fue un socialista convencido, compartía con Dewey su desdén por cierto determinismo marxista, entendido como la exclusión de cualquier factor no-económico en el devenir de las sociedades y expresado en el célebre lema «la infraestructura determina la superestructura». Según Neurath,

"sería un error pensar que las enseñanzas de Manchester sobre la competencia, la doctrina del laissez-Jaire, fueron la causa del cambio, pero sería igualmente erróneo decir que el cambio, que en cualquier caso se estaba produciendo en todas las áreas, era la causa de esas enseñanzas. Sería mucho mejor decir que esas enseñanzas junto con otras condiciones fueron causas del curso entero de los acontecimientos, ya que en general prevalece la intuición básica de que la totalidad de lo que ocurre en un momento del tiempo es causa de lo que ocurre en el siguiente. Debemos evitar concebir una parte de esos acontecimientos como causa de otra parte, los procesos productivos como causas de lo religioso, lo moral o lo políticon ${ }^{27}$.

Dewey coincide plenamente con Neurath en este punto. De hecho, el determinismo económico ejemplificaría un tipo de falacia filosófica contra la que su pragmatismo es buen antídoto: convertir un factor que conocemos gracias a un proceso de indagación en la condición antecedente y causal de todo ese proceso. Con todo, admite Dewey, en la formulación marxista original,

«se hacía una importante distinción que las posteriores manifestaciones han tendido a pasar por alto, pues se admitía que una vez producidas las relaciones políticas, científicas, etc., obran como causas de fenómenos subsiguientes y que, en esta capacidad, pueden modificar en cierto grado la operación de las fuerzas que originalmente la produjeron" (LW. 13.11 S).

Pero de hecho, según Dewey, el determinismo económico pierde todo su mordiente una vez admitida la distinción: nos vemos obligados a reconocer que la única manera de determinar qué factores se deben a la ciencia, por ejemplo, y qué factores obedecen exclusivamente a la producción económica sería

27 Citado por CARTWIRGHT, o.c., p. 227. 
la investigación científica. Como hemos visto, admitir la ciencia para investigar la ciencia es el rasgo distintivo de la epistemología naturalizada que, mucho antes que Quine, Dewey y Neurath compartían.

La participación de Neurath en la BildungPolitik del ayuntamiento socialista de Viena y la intensa actividad educativa e institucional de John Dewey en Chicago son buena muestra del papel que concedían a la ciencia como uno de los motores del cambio social. Como ya adelantaba, el papel concedido a la educación científica es el motivo que finalmente impulsa la colaboración entre Dewey y Neurath en la Enciclopedia de la Ciencia Unificada. Pero sostener una concepción de la ciencia como instrumento para la transformación de la realidad pública significa para Dewey y Neurath contraer un compromiso con la educación como agente del cambio social. A su vez, ese compromiso explica los numerosos proyectos enciclopédicos y pedagógicos en que Dewey y Neurath se embarcaron. Además de la Enciclopedia de la Ciencia Unificada, Neurath impulsó en un tesauro visual, un compendio de educación visual, un compendio de estadística gráfica (el denominado método de Viena, completado más tarde con el proyecto de isotipos), aparte de participar en el diseño del los museos industriales de las ciudades de Chicago y de México. Dewey, por su parte, participó en un diccionario de la psicología y la filosofía, un diccionario de la educación, una enciclopedia de la educación y en una enciclopedia de las ciencias sociales, entre otros numerosos proyectos.

Etimológicamente, "Ciclo-Paedia" hace referencia al ciclo educativo, como el trivium (gramática, dialéctica, retórica) y el quadrivium (aritmética, geometría, astronomía y música) del medioevo europeo. El motivo educativo de la Enciclopedia de la Ciencia Unificada es afin al de la Enciclopédie francesa de Diderot y D'Alambert: la educación como instrumento de emancipación. En una carta a Neurath, Einstein confiaba en que la Enciclopedia Internacional de la Ciencia Unificada de Neurath cumpliese la misma función que la Enciclopédie francesa desempeñó durante la Revolución Francesa ${ }^{28}$. Y resulta significativo que Dewey rescate el motivo de la educación para la emancipación como elemento positivo del empirismo clásico:

«En su lado positivo, la filosofía empirista fue empleada para realzar la importancia de la educación [...] [los pensadores franceses] afirmaban que 
es posible construir cualquier tipo de mente o carácter controlando las impresiones, las sensaciones y las observaciones de esa mente, y sobre todo controlando las asociaciones formadas con la suma de placer o dolor [...] En su lado positivo, el empirismo era pues un ideal asociado con el concepto dieciochesco de progreso y con la visión abierta a la infinita perfectibilidad de la humanidad, una vez erradicada la corrupción procedente de instituciones nefastas, políticas y eclesiásticas, dando oportunidad a la educación y a la racionalidad" (LW. 11.80)

Por mal que suene a nuestros oídos posmodernos, tanto Neurath como Dewey compartían a grosso modo este ideal ilustrado, pero ya desprovisto de todo elemento finalista. La noción de progreso era para ambos una apuesta por la acción social y no un marco teleológico desde el que justificar la racionalidad de lo real. $Y$ es precisamente la adhesión a ese ideal ilustrado el que justifica el carácter radicalmente anti-metafísico del pensamiento de ambos autores.

Para Neurath, la lucha contra la metafísica tenía un carácter más político que epistémico. Se trataba de la lucha contra todo tipo de autoritarismo, entendido como la creencia en todo tipo de sistema pre-establecido, esto es, que no sea fruto de los esfuerzos de cooperación humanos y que, por tanto, no esté siempre abierto a ulteriores cambios. En cierto sentido, la lucha contra las aserciones metafísicas es también para Dewey la lucha contra ideales absolutos para los que no se establecen condiciones de producción efectiva. Para Dewey

"La pretensión de estar en posesión de verdades últimas no es sino un llamamiento a que sea la fuerza el elemento último de arbitraje» (LW1).

Para Neurath y Dewey, luchar contra la metafísica era un modo de combatir la incondicionalidad y, por lo tanto, de apostar por la negociación.

La demarcación cognitiva entre ciencia y metafísica se transforma así en una demarcación explícitamente social. Si los problemas de la metafísica son pseudoproblemas no es porque no sean ni empíricos ni conceptuales, como pretendía el positivismo lógico, sino por su artificialidad. Según Dewey, la actitud anticientífica es aquella que, en vez de afrontar los problemas que surgen de la interacción entre las sociedades humanas y sus respectivos entornos biológicos y culturales, los suplanta por problemas artificiales y las soluciones existentes de antemano. 
«Artificiales son los problemas que no surjan de las condiciones bajo las cuales se desarrolla la vida... El hogar, la escuela, la tienda, el lecho y el hospital presentan problemas tan genuinos como los laboratorios, y habitualmente de manera más directa y urgente [...] si entendemos por metafísica aquello que está fuera de la experiencia, por encima y allende ésta, todos los seres humanos son metafísicos cuando se ocupan de problemas que no surgen de la experiencia y de soluciones que están fuera de ésta. Los hombres son aún metafísicos no sólo en un sentido técnico, sino sobre todo cuando se ocupan de moral y de política» (LW. 13.273-274).

Dewey tiene razón cuando afirma que dejar que el método experimental substituya a la fe o la tradición en el ámbito del conocer e impedir que lo haga en otros ámbitos es la manera de que los beneficios de ese método se instrumentalicen para perpetuar intereses de clase, prejuicios nacionales, raciales o de género en la conducción pública de los asuntos humanos. Es aquí donde el problema de la unificación de la ciencia muestra su significación social. Para Dewey y para Neurath, el buen gobierno de los asuntos públicos depende de la correlación sistemática de predicciones pertenecientes a distintos campos humanos: desde la meteorología a la economía, desde la sociología a la geología, desde la biología a la termodinámica.

En nuestros días, la idea de que esa sistematización sea factible, o siquiera deseable, cuenta con muchos menos adeptos que en los tiempos de Dewey y Neurath. Pero tampoco creo que ninguno de los dos fuera demasiado ingenuo al respecto. En estas páginas he reiterado la desconfianza que ambos mostraban hacia la noción filosófica de sistema. Más próximo al enciclopedismo y a su espíritu cooperativo se encuentra la idea de Neurath de que la unidad de la ciencia es análoga a la unidad de un mosaico ${ }^{29}$. Para acabar, desarrollaré brevemente esta analogía de Neurath. Curiosamente, dicho sea de paso, creo que fue William James uno de los primeros en emplear la analogía del mosaico en su conferencia "Un Universo Pluralista". La suya, afirma James, es una filosofía de mosaico porque pone el énfasis explicativo en la parte, el elemento, el individuo. Un énfasis que resulta bastante distinto de la significación social que venimos atribuyendo al lema de la unificación de la ciencia en Dewey y

29 Otto Neurath, "The New Encyclopedia», en B. MacGuiness, (ed.) Unified Science (Dordrecht: Reidel, 1987), p. 136; Véase también "Unity of Science as Enciclopedic Integration", en en Philosophical Papers, 1913-1946 (Dordrecht: Reidel, 1983). 
Neurath. Con todo, creo que es posible interpretar esta última dándole un aire más acorde con un pragmatismo social y cooperativo. De hecho, Neurath plantea la analogía para poner énfasis en la continuidad o unidad diacrónica en la historia de la ciencia: la historia de un mosaico y de las generaciones que aportan sus teselas ${ }^{30}$.

Una de las posibles imágenes que evoca la analogía del mosaico es la del puzzle o rompecabezas. Con todo, la unidad del mosaico no es comparable con la unidad del rompecabezas de la ciencia normal kuhniana, un puzzle aparentemente reductible a piezas y conjuntos de piezas hechas de antemano que el practicante ha de saber ensamblar para demostrar su competencia como experto. El mosaico de Neurath es un vasto diseño, con teselas que se deterioran, caen y son reemplazadas por otras, nunca idénticas a las anteriores, y cuyo acomodo implica la renovación de grandes áreas del mosaico. Pero como bien dijo Kuhn y bien saben los arqueólogos, en alguna ocasión puede aparecer una pieza tan anómala que quede arrinconada, pues nadie sabe muy bien qué hacer con ella. Otras veces, para preservar algunas teselas, es preciso aislarlas, colocando en torno suyo un anillo de piezas creadas ad hoc y que suelen provocar serias discontinuidades con el resto del patrón. Un patrón que a veces crece, pero no uniformemente, a menudo en unas direcciones y no en otras, que habrán de esperar tiempos mejores. En ocasiones, el ardor de algún epistemólogo logra convencernos para que arranquemos grandes partes del mosaico, declarándolas inválidas. Con todo, esas áreas siempre muestran una insistente tendencia a reaparecer, incluso en zonas adyacentes a otras áreas del mosaico que casi siempre han gozado de una conservación privilegiada.

Creo que, en el fondo, las teselas del mosaico de Neurath son como las planchas de su célebre barco. Por eso debemos guardamos de concebir su mosaico como un constructo que, en última instancia, duplica, refleja o representa, pero que es absolutamente separable del mundo. Dicho sea en términos más deweyanos, el mosaico es la experiencia humana históricamente entendida: nuestra experiencia común transformada, hecha transitable a través de las generaciones.

30 Unity of Science as Encyclopedic Integration", en en Philosophical Papers, 1913-1946 (Dordrecht: Reidel, 1983). 